\title{
A Scalar Field as a Candidate for the Cosmological Non-baryonic Dark Matter
}

\author{
Hernando Quevedo, ${ }^{1}$ Marcelo Salgad o $^{1}$ and Daniel Sudarsky ${ }^{1}$
}

Received October 26, 1998

\begin{abstract}
We present an up-to-date report on the status of the cosmological model based on a massive scalar field non-minimally coupled to gravity, which has been recently used to explain the apparent periodicity in the distribution of galaxies, and as a model for the missing non-baryonic component of dark matter, within the standard inflationary scenario $(\Omega=1)$. The model agrees with most cosmological observations, however local experiments can pose serious constraints that indicate the necessity of a slightly modified model.
\end{abstract}

\section{INTRODUCTION}

It is by now widely accepted that the luminous matter represents only a tiny fraction of the matter content of the Universe. The evidence is found in the dynamics of galaxies [1], in the behavior of clusters of galaxies [2], and finally, from the so-called inflationary scenarios [3] that indicate that $\Omega_{\text {total }}=1$ (this prediction can be avoided by fine-tuning and/ or complicating the model [4] and we will not consider this possibility). This matter must be mostly exotic matter, as it cannot consist of radiation or baryonic matter since the primordial abundance of light elements $(\mathrm{Be}$, $\mathrm{Li}, \mathrm{D}$, etc) restricts the value of the baryonic energy density $0.007 h^{-2}<$ $\Omega_{\mathrm{bar}}<0.024 h^{-2}[5,6]$, where $h$ is the Hubble constant in units of 100 $\mathrm{km} \mathrm{s}^{-1} \mathrm{Mpc}^{-1}$, with likely values in the range [0.65,0.75] (see, for instance, Refs. 7,8).

Instituto de Ciencias Nucleares, Universidad Nacional Autónoma de México, A. P. 70-543 México 04510 D.F, México 
Moreover most of the dark matter must be distributed homogeneously [9] since the most recent dynamical determinations of the amount of matter in galaxies, galaxy clusters and superclusters put $\Omega_{\mathrm{dyn}}=0.3 \pm 0.1$ [10]. Note that this indicates that it is very likely that some of the dynamical dark matter (as opposed to the homogeneous component that does not affect local dynamics) is non-baryonic as well [11]. We will not consider this last point in our initial analysis. Furthermore the small anisotropy in the temperature of the Смв (cosmic microwave background) $\Delta T / T \approx$ $10^{-5}$ puts severe limits on the degree of clumpiness that can be present at each scale. This arises through the Sachs-Wolfe effect [12] (a result of the time dependence of the density perturbations) which yields for a perturbation of size $L$ and density fluctuation $\delta \rho / \rho$ the estimate $\Delta T / T=$ (1/2) $\delta \rho / \rho\left(H_{0} L\right)^{2}$. Cosmologists and particle physicists have long been considering what this exotic matter might be. The main models can be divided in two categories: hot dark matter (relativistic at the time of structure formation) and cold dark matter (non-relativistic at the time of structure formation). The latter is the preferred one in the light of the theory of structure formation [13]. No independent evidence for the existence of these new types of matter has been found so far.

An apparent ly completely independent problem in cosmology is posed by the recent observations in deep pencil beam surveys [14,15], showing that the galaxy number distribution exhibits a remarkable periodicity. This would be catastrophic for our cosmological conceptions. Another, probably unconnected, puzzling observation indicates quantized redshifts at a much smaller scale [16]. While it is true that such periodicity has been observed only in a couple of the few directions that have been explored so far [17], it would be a remarkable coincidence if it turns out that it is absent in most other directions, and we just happened to have chosen to explore one of the few directions in which that phenomena can be observed. It seems, therefore, reasonable to assume that the periodicity will be also present in the deep pencil beam surveys in most other directions [22]. We must point out that on top of this periodic structure we also expect a standard and more random structure to be present that could explain the absence of the periodicity in some directions [18] The issue is of course whether the periodicity is present in most directions. The expected presence of the more random structure indicates that more weight should be given to the deeper surveys that are less likely to miss the suggested underlying periodic structure. Moreover, the alternative possibility, namely that a real non-periodic structure exists to the extent indicated in [15] with scale of the order of $100 \mathrm{Mpc}$ seems very difficult to reconcile with the bounds arising from the isotropy of $T_{\mathrm{CMB}}$ in view of the Sachs-Wolfe 
effect [19].

The only known explanation for such periodicity that is compatible with the cosmological principle is to assume that there is only an apparent spatial periodicity which is the result of a true temporal periodicity which shows up in our observations of distant points in the Universe, and which is mistakenly interpreted as a spatial periodicity [20,24]. Various specific models that have been proposed [20,21,24] have been either dismissed [25] or shown to have serious problems [30]. In this light, the Oscillating $G$ Model seems to be the most attractive alternative. We note that in the fossil record of marine bivalve shells [31], there is further evidence for an effective gravitational constant that oscillates with time.

In this work, we consider the model, initially proposed in [24], of a massive scalar field non-minimally coupled to gravity leading to an effective gravitational constant which oscillates in cosmic time. We will consider the cosmological non-baryonic component of dark matter, modeled by the scalar field and will not deal with the local dark matter problem which is presently under investigation.

\section{THE MODEL}

The main feature of the model is a homogeneous scalar field $\phi$ with a non-minimal coupling to gravity. One of the simplest models is described by the Lagrangian density,

$$
\mathcal{L}=\left(\frac{1}{16 \pi G_{0}}+\xi \phi^{2}\right) \sqrt{-g} R-\sqrt{-g}\left[\frac{1}{2}(\nabla \phi)^{2}+m^{2} \phi^{2}\right]+\mathcal{L}_{\text {mat }} .
$$

Here $G_{0}$ is the Newtonian gravitational constant, $\xi$ is a coupling constant, and $m$ is the mass associated with the scalar field $\phi$. In this model we are also including a schematic matter Lagrangian $\mathcal{L}_{\mathrm{m}}$ at. Equation (1) shows that the introduction of the coupling term corresponds to an effective gravitational constant which explicitly depends on the scalar field, $G_{\text {eff }}=$ $G_{0} /\left(1+16 \pi G_{0} \xi \phi^{2}\right)$.

We consider a FRW Universe with $k=0$ as dictated by the standard inflationary models. For the energy-momentum tensor of ordinary matter we consider a combination of two non-interacting perfect fluids, the baryonic matter and the radiation field.

Our analysis consists in evolving the scale factor, the scalar field and the ordinary matter densities backwards and forwards in cosmic time. For given values of the model parameters $\xi$ and $m$, the data corresponding to today's values of $H_{0}, \Omega_{\mathrm{bar}}, \Omega_{\mathrm{rad}}, \phi_{0}$ and $\dot{\phi}_{0}$ is what is needed to integrate 
the field equations numerically. For simplicity we set $\dot{\phi}_{0}=0$ to be in agreement with the bound $\dot{G} /(G H) \leq 0.3 h^{-1}$ [21].

One of the main problems that are faced by this type of models is that related to the primordial nucleosynthesis of ${ }^{4} \mathrm{He}$. The latter is determined by the temperature $T_{E}$ at which the rate of weak interactions, $\Gamma_{w k}(T)$, equals $H(T)$. According to standard cosmology, the value $T_{E}=0.7 \mathrm{MeV}$ is in good agreement with observational data [33]. The models, in which $G_{\text {eff }}$ is allowed to vary can, in principle, produce very different values for the ${ }^{4} \mathrm{He}$ abundance. In the present case, the evolution of the scalar field backwards in cosmic time results in it going to $\pm \infty$ or stabilizing near zero (a situation we have called "the plateau") depending on the precise initial data (i.e., the data corresponding to today's Universe). Thus we assume that there is some mechanism (probably related to inflation) that drives the scalar field from an arbitrary value near the Big Bang to an extremely low value before the era of nucleosynt hesis (we have called this assumption the plateau hypothesis). Our calculations show that the field will remain at that value up to and beyond the nucleosynthesis era. So we will have $G_{\text {eff }} \approx G_{0}$; i.e., once in this plateau the field $\phi$ will remain there to be later "amplified" by the curvature coupling, precisely before the onset of oscillat ory behavior (when $H \approx m$ ) [26,27]. Therefore, a "fine-tuning" of the initial data guarantees that the scalar field is practically zero and thus $G_{\text {eff }} \approx G_{0}$ during nucleosynthesis. We note, however, that this is not the kind of fine-tuning that implies the dismissal of the model, but it is rather a procedure that becomes necessary in order to obtain the data describing our Universe today if it did go through this "plateau" phase. The highly nontrivial question is whether those data are compatible with current observations [30].

In order to carry out this analysis, the constants $\Omega_{\mathrm{bar}}$ and $\Omega_{\mathrm{rad}}$ are fixed by the "initial" conditions which we choose as their value at present time $\left(t_{0}\right)$. In particular, $\Omega_{\mathrm{rad}}$ corresponds to $T_{\mathrm{CMB}}=2.73 \mathrm{~K}$. The total energy density of the Universe is $\Omega=\Omega_{\mathrm{bar}}+\Omega_{\mathrm{rad}}+\Omega_{\phi}=1$. Thus fixing $\Omega_{\text {bar }}$ is equivalent to fixing $\Omega_{\phi}$. The strategy of our analysis consists in starting from a specific value of the baryonic energy density (i.e. one that lies within the range allowed by nucleosynthesis) and the frequency of the periodicity, and then we look for the value of the oscillation amplit ude $\mathcal{A}_{0}$ that is compatible with the plateau hypothesis. We then ask whether this allows the required value $\mathcal{A}_{0} \approx 0.5$ [20]. This is a nontrivial question and we have shown that the answer is affirmative [30]. Moreover, these values, together with the value of the Hubble parameter, determine a specific value for the age of the Universe, $T_{U}$, and so it is a highly nontrivial question whether these results are or are not compatible with the rather 
conservative limit $T_{U}>11.5 \mathrm{Gyr}$ [29]. Although recent observations [28] of objects at a redshift of 7 suggest even larger values. In [30] we have shown the compatibility of the model with observational data, provided that $h<0.68$.

Note that in this model most of the energy density of the Universe resides in the oscillating scalar field. Consequent ly, $\Omega_{\phi}$ can be interpreted as the energy density of the previously unseen cosmological dark matter that would have been detected indirectly through its effect on $G_{\text {eff }}$.

The model satisfies the requirements expressed in the initial considerations in that the most important component of matter in the Universe is non-baryonic in nature, and be distributed homogeneously. Thus the problems of the nature of cosmological dark matter and of the periodicity in the galaxy number distribution may be solved simultaneously within the framework of an oscillating $G$ model with a massive scalar field.

On the other hand, the analysis of the local issues indicates that the model might need some modifications (see next section).

\section{LOCAL CONSTRAINTS}

It is well known that the Viking radar experiments [32] impose severe constraints on the Brans-Dicke parameter $\omega_{\mathrm{BD}}>500$ (at $1 \sigma$ level) and therefore that any scalar-tensor theory with an effective non-constant parameter $\omega_{\mathrm{BD}}^{\text {eff }}(\phi)$ can only be considered as a potentially successful candidate for a theory of gravity if it passes such a limit. Moreover, the Solar System experiments also constrain the variation in $G$ by the limit $\dot{G} /(G H) \leq 0.3 h^{-1}$.

The model described by (1) contains a few parameters (see Refs. 27,30) all of which are determined by our previous cosmological analysis. That is, there are more observational bounds than parameters in (1). For instance the bound $\dot{G} /(G H) \leq 0.3 h^{-1}$ has been avoided by assuming the initial condition in the scalar field as $\dot{\phi}_{0}=0$. On the other hand, the inflationary condition $\Omega=1$, the bound $0.007 h^{-2} \leq \Omega_{\mathrm{bar}} \leq 0.024 h^{-2}$ imposed by the abundances of ${ }^{3} \mathrm{He}, \mathrm{Be}$, Li, etc., the nucleosynthesis of ${ }^{4} \mathrm{He}$, and the observed apparent periodicity of $128 \mathrm{Mpc} h^{-1}$ in the galaxy number density which fixes the mass $m \sim 10^{-31} \mathrm{eV}$ completely determine the other parameters $\xi$ and $\phi_{0}$ of the model [26,27]. This implies that with the above assumptions the model, if viable, should also pass the limits on the red-shift-galaxy amplitude $0.5 \leq \mathcal{A}_{0}$, the age of the Universe, and the one on $\omega_{\mathrm{BD}}^{\mathrm{eff}}(\phi)$. In previous papers $[27,30]$, we showed that the model is in agreement with the first two, whereas with the values obtained for $\xi(\sim 6)$ and $\phi_{0}\left(\sim 10^{-3}\right)$ the model is unable to satisfy the Brans-Dicke test. That 
is, the model (1) and the Brans-Dicke limit imply that

$$
\omega_{\mathrm{BD}}^{\mathrm{eff}}(\phi)=\frac{1+16 \pi \xi \phi^{2}}{8 \cdot 16 \pi \xi^{2} \phi^{2}}>500 .
$$

Figure 1 shows the region allowed by the above limit in the $\xi-\phi_{0}$ plane. The shadow region which is forbidden contains the set of points that emerged from the analysis of the model (1) in the cosmological setting.

Several possibilit ies arise that might allow the model to overcome the problem put by the limits on $\omega_{\mathrm{BD}}^{\mathrm{eff}}(\phi)$. One is the relaxation of the condition $\dot{\phi}_{0}=0$ to its maximum value allowed by the limit $\dot{G} /(G H) \leq 0.3 h^{-1}$. This could in principle reduce the values of $\xi$ and $\phi_{0}$, and thus increase $\omega_{\mathrm{BD}}^{\mathrm{eff}}(\phi)$.

Another possibility is to consider the changes of the value of $\phi_{0}$ in the Solar System neighborhood with respect to its cosmological value caused by local effects. After all, the field will oscillate inside the galaxy with a mass-frequency $m_{\text {eff }}^{2}=m^{2}+8 \pi G_{0} \rho \xi \approx m^{2}\left[1+12 \xi h^{-2}\left(\rho_{\text {galaxy }} / 10^{-23} \mathrm{~g} \mathrm{~cm}^{-3}\right)\right]$ that differs from the cosmological mass-frequency $m$ [30].

Another alternative consists in changing $\phi_{0}$ due to a mechanism similar to that of "scalarization" in neutron stars [34,35].

We should mention that the analysis of the binary-pulsar data,within the context of this type of theory, indicates a constraint on the value of $\xi$, namely $\xi<2.5$. However, such an analysis uses a particular choice of the equation of state for the nuclear matter (polytrope), and it is not clear which changes might arise from the use of more realistic equations of state.

Finally, let us comment that the introduction of a scalar potential of the kind $m^{2} \phi^{2}+\lambda \phi^{4}$ could serve to ameliorate this problem by adjusting the correct value of $\lambda$. This modification has already been suggested on different grounds [21]. All these issues are currently under investigation.

\section{DISCUSSION}

To conclude, the oscillating $G$ model is certainly the most attractive model for explaining the observed periodicity in the galactic distribution, and it should also be considered as a cosmological dark matter model which is, however, indirectly observable in the oscillation of the galactic distribution. Although other models are still under consideration, they are based on specific structures (fractal, pancake-like, etc.) for the distribution of matter in the Universe [19].

The initial analysis of the local aspects of the theory indicates that some changes must be made in the model in order to satisfy the constraints arising from the binary pulsar and the Solar System. The required adjustments correspond to factors of order 2 or 3 so it is by no means a hopeless 


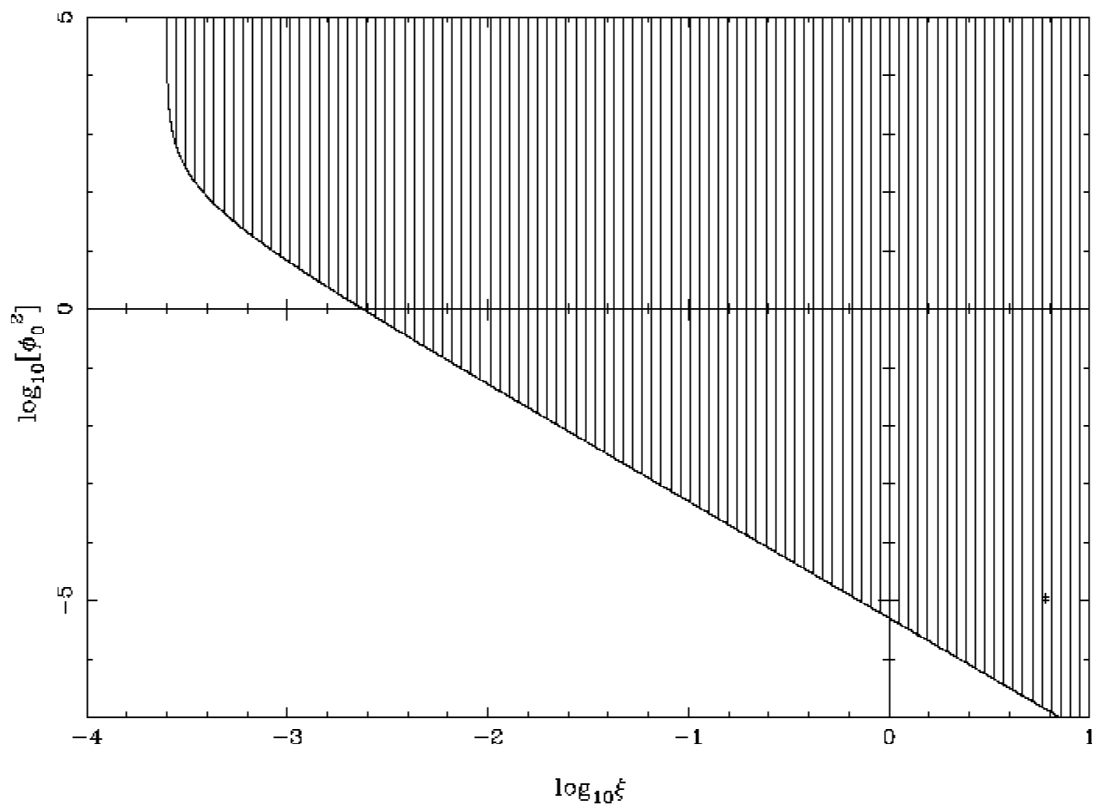

Figure 1. Limits imposed by the Viking experiments on the parameters $\phi_{0}^{2}$ and $\xi$ of the oscillating $G$ model. The shadow region is forbidden by observations, and the asterisk depicts a representative set of values that however succeed in passing the most important cosmological bounds.

enterprise, and if the existence of periodicity in most directions is confirmed, this model would be the most attractive explanation.

\section{REFERENCES}

1. Van Albada, T. S., Bahcall, J. N., and Begeman, K. (1987). In Dark Matter in the Universe (Proc. Jerusalem Winter School for Theoretical Physics), vol. 4, J. Bahcall, T. Piran, and S. Weinberg, eds. (World Scientific, Singapore).

2. Ostriker, J. P., and Thompson; C., Tremane, S., and Lee, H. M. (1987). In Dark Matter in the Universe (Proc. Jerusalem Winter School for Theoretical Physics), vol. 4, J. Bahcall, T. Piran, and S. Weinberg, eds. (World Scientific, Singapore).

3. Guth, A. H. (1981). Phys. Rev. D23, 347; Linde, A. (1982). Phys. Lett. B108, 389.

4. Bucher, M., Goldhaber, A. S., and Turok, N. (1995). Phys. Rev. D52, 3314; Linde, A. D., and Mezhlumian, A. (1995). Phys. Rev. D52, 6789; Gott, J. R. (1992). Nature 295, 304.

5. Copi, C., Schramm, D. N., and Turner, M. S. (1995). Science 267, 192.

6. Ostriker, J. P., and Steinhardt, P. J. (1995). Nature 377, 600.

7. Friedman, W. L., et al. (1994). Nature 371, 757.

8. Riess, A. G., Press, W. H., and Kirshner, R. P. (1995). Astophys. J. 438, L17.

9. Turner, M. S., and White, M. (1997). Preprint astro-ph / 9701138. 
10. Trimble, V. (1987). Ann. Rev. Astron. Astrophys. 25, 425; Dekel, A., Burstein, D., and White, S. (1996). Preprint astro-ph/ 9611108.

11. Fukugita, M., Hogan, C. J., and Peebles, P. J. E. (1996). Nature 381, 489.

12. Sachs, R. K., and Wolfe, A. M. (1967). Astrophys. J. 147, 73.

13. Blumenthal, A., et al. (1984). Nature 311, 517.

14. Broadhurst, T., Ellis, R., Koo, D., and Szalay, A. (1990). Nature 343, 726; Szalay, A., Ellis, R., Koo, D., and Broadhurst, T. (1991). In Primordial Nucleosynthesis and Evolution of the Universe, K. Sato and J. Audouze, eds. (Kluwer Academic, Dord recht) p. 435.

15. Szalay, A., Broadhurst, T.J., Ellman, N., Koo, D., and Ellis, R. (1993). Proc. Natl. Acad. Sci. USA 1993, 4853.

16. Matthews, R. (1996). Science 271, 759.

17. Willmer, C. N. A., et. al. (1994). Astrophys. J. 437, 560 .

18. Bellanger, C., De Lapparent, V. (1995). Astrophys. J. 455, L1; Ettori, S., Guzzo, L., and Terenghi, M. (1998). Mon. Not. R. Astr. Soc., in press; Cohen, J. G., et. al. (1996). Preprint astro-ph/9608121.

19. Labini, F. S., Montuori, M., and Pietronero, L. (1998). Phys. Rep. 293, 61.

20. Hill, C. T., Steinhardt, P. J., and Turner, M. S. (1990). Phys. Lett. B252, 343.

21. Crittenden, R. G., and Steinhardt, P. J. (1992). Astrophys. J. 395, 360.

22. Bundich, P., Nurowski, P., Raczka, R., and Ramella, M. (1995). Astrophys. J. 451, 10.

23. Hill, C., Steinhardt, P. J., and Turner, M. (1991). A strophys. J. 366, L57.

24. Morikawa, M. (1990). Astrophys. J. 362, L37.

25. Sudarsky, D. (1992). Phys. Lett. B281, 98.

26. Salgado, M., Sudarsky, D., and Quevedo, H. (1996). Phys. Rev. D53, 6771.

27. Salgado, M., Sudarsky, D., and Quevedo, H. (1997). Phys. Lett. B408, 69.

28. Vogel, G. (1996). Nature 274, 2006.

29. Jimenez, R., et al. (1998). Mon. Not. R. Astron. Soc., in press.

30. Quevedo, H., Salgado, M., and Sudarsky, D. (1997). Astrophys. J. 487, 14.

31. Sisterna, P. D., and Vucetich, H. (1994). Phys. Rev. Lett. 72, 454.

32. Reasenberg, R. D., et al. (1979). Astrophys. J. 234, L219.

33. Kolb, E. W., and Turner, M. S. (1990). The Early Universe (Addison-Wesley, Redwood City).

34. Damour, T., and Esposito-Farese, G. (1993). Phys. Rev. Lett. 15, 2220.

35. Damour, T., and Esposito-Farese, G. (1996). Phys. Rev. D54, 1474. 